\title{
INTEGRAL long term monitoring of 4U 1722-30: evidence for spectral state variations
}

\author{
Antonella Tarana ${ }^{* \dagger}$ \\ IASF-Rome/INAF, via Fosso del cavaliere 100, 00133 Rome, Italy \\ E-mail: antonella.tarana@iasf-roma.inaf.it
}

\section{Angela Bazzano}

IASF-Rome/INAF, via Fosso del cavaliere 100, 00133 Rome, Italy

\section{Memmo Federici}

IASF-Rome/INAF, via Fosso del cavaliere 100, 00133 Rome, Italy

\section{Pietro Ubertini}

IASF-Rome/INAF, via Fosso del cavaliere 100, 00133 Rome, Italy

\begin{abstract}
We report on the 2003-2005 INTEGRAL observations of the Neutron Star Low Mass X-ray Binary 4U 1722-30 (also known as GRS 1724-30) located in the Globular Cluster Terzan 2. The JEM-X and IBIS light curves show the source with a persistent yet variable flux. The Hardness-Intensity diagrams highlight the behaviour of a typical Atoll source: 4U 1722-30 repeatedly moves in the diagrams from the Banana (Soft state) to the Island (Hard state). We report on the detailed spectral analysis of Soft and Hard states and, for the first time, also of an Intermediate state. The Hard spectra reveal a Comptonised corona emission up to $200 \mathrm{keV}$ characterized by a high temperature of $40 \mathrm{keV}$ and optical depth of 0.5. In the Soft state the main emission is from the accretion disk (with $\mathrm{kT}_{\text {in }} \sim 0.5 \mathrm{keV}$ ) whereas the Comptonised emission decreases showing an optically thick and cold corona $\left(\tau \sim 9, \mathrm{kT}_{\mathrm{e}} \sim 2 \mathrm{keV}\right)$. During the hardening there is an increase of the inner radius of the accretion disk suggesting a system expansion during the spectral transition. This behaviour is reminescent of the Soft X-ray transient sources though 4U 1722-30 never reaches a real "quiescent" state.
\end{abstract}

7th INTEGRAL Workshop

September 8-112008

Copenhagen, Denmark

* Speaker.

${ }^{\dagger}$ This work has been supported by the Italian Space Agency through the grant ASI/INAF I/008/07 and I/008/06. 


\section{Introduction}

4U 1722-30, also known as GRS 1724-30, is a bright LMXB located in the Globular Cluster Terzan 2 [1]. The observed Type $1 \mathrm{X}$-ray bursts indicate that the compact object is a weakly magnetized neutron star [1], [2]. The timing properties outlined with RXTE observations suggest that its timing properties are typical of an atoll source [3]. 4U 1722-30 is a persistent though variable source, and it is one of the first neutron star systems from which hard X-ray emission (E $>35 \mathrm{keV}$ ) was detected by SIGMA with a power law spectrum with photon index $\Gamma \sim 1.65$ extending above $100 \mathrm{keV}$ [4]. Previous EXOSAT observation didn't reveal flux above $10 \mathrm{keV}$ [5]. BeppoSAX and RXTE allowed a broad band observations, detecting the source with a Comptonized spectrum extending up to $200 \mathrm{keV}$, plus an additional soft componenet (below $3 \mathrm{keV}$ ), described by a blackbody emission.

\section{The INTEGRAL monitoring}

We monitored the sources with INTEGRAL during the period October 2003 - April 2005, collecting a total of 883 pointing for IBIS and 256 pointings for JEM-X. Because of this long term monitoring, INTEGRAL detected the source in different spectral states and for the first time a detailed spectral analysis of different spectral states has been performed.

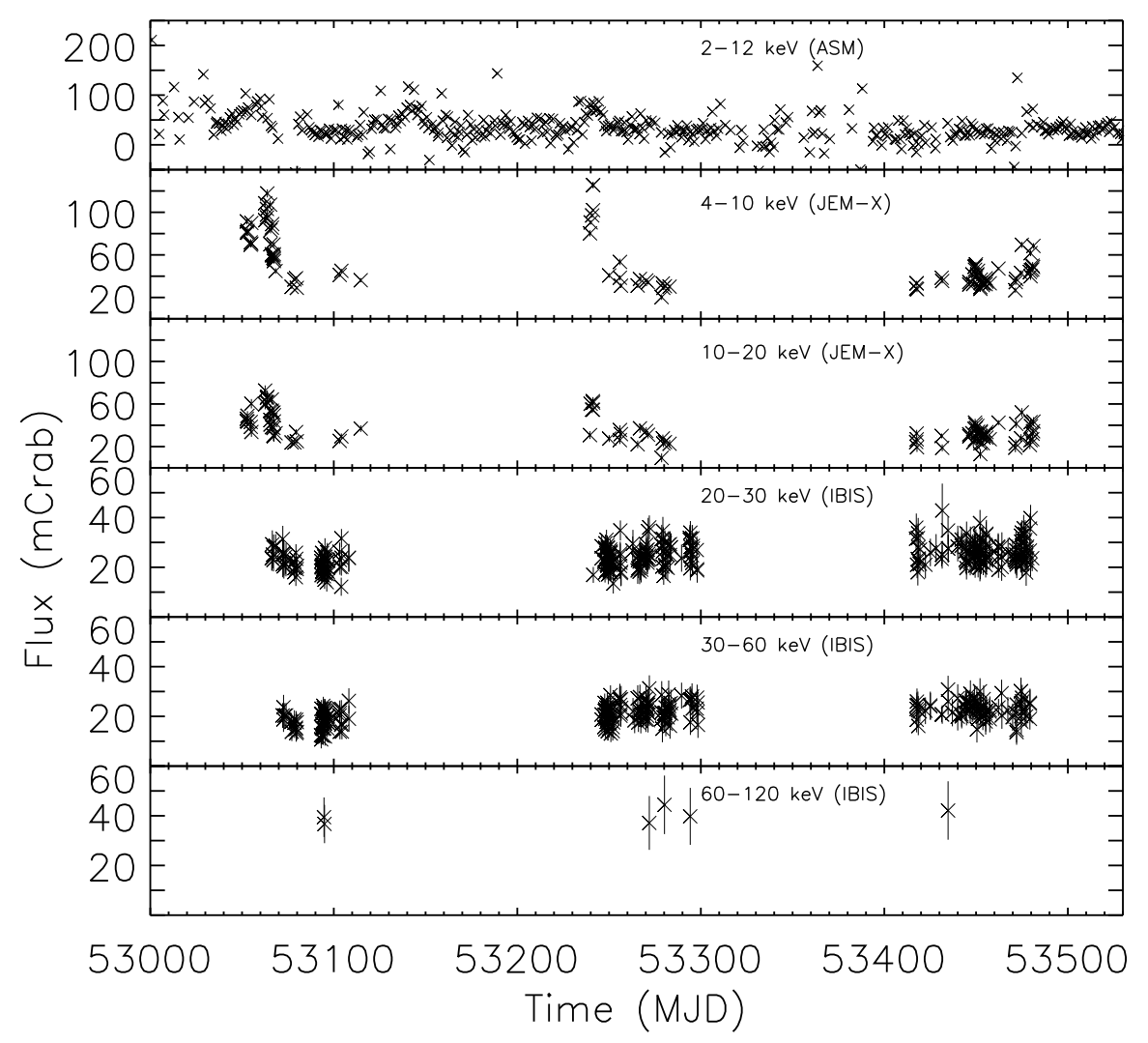

Figure 1: INTEGRAL and ASM/RXTE light curves of 4U 1722-30. 

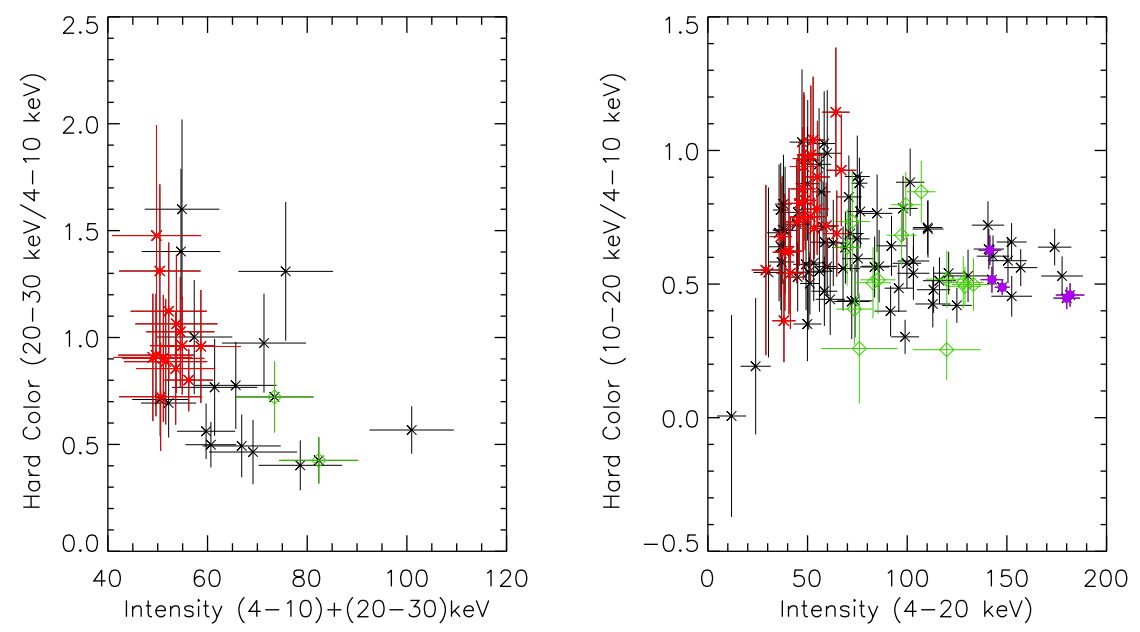

Figure 2: Left: IBIS-JEM-X Very hard color-intensity diagram. Right: JEM-X hard color-intensity diagram. The colors identify the spe1 (purple), spe2 (green) and spe3 (red) data sets (see Table 1).

\section{The light curves and hardness-intensity diagrams}

The JEM-X and IBIS light curves in different energy bands are shown in Figure 1. Each INTEGRAL point correspond to a single pointing lasting about 2000 seconds. In the top panel are reported the ASM/RXTE one-day monitoring during the same period. The source reveals a flux variation (similar to outbursts) in the soft band $(<20 \mathrm{keV})$, while in the hard ones $(>20 \mathrm{keV})$ it is more rarely significantly detected and the flux changes to a minor extent.

We constructed two hardness-intensity diagrams, the first one with the JEM-X data and the second one with IBIS and JEM-X simultaneous data. Both are shown in Figure 2, with indication on the Intensity and Color bands used. The source moves through the diagram showing spectral changes. We show with different color the different spectral data sets for which observations log is reported in Table 1. The purple data set refers only to the JEM-X/JEM-X hardness intensity diagram because of the lack of high energy detection by IBIS. This data set correspond in fact to a Soft (banana) spectral state. The green and red pointings correspond to a hardening of the sources that enters the Hard (island) spectral state. These pointings are reported in both the hardnessintensity diagrams. The mass accretion rate increases during the softening with a corresponding decreasing of the inner accretion disk radius (see discussion for details).

Table 1: The $\log$ of the INTEGRAL/IBIS and JEM-X data used for spectral fitting of 4U 1722-30.

\begin{tabular}{llll}
\hline Data set & Time start (MJD) & $\begin{array}{l}\text { Exposure }(\mathrm{ks}) \\
\text { IBIS } ; \text { JEM-X }\end{array}$ & Spectral state \\
\hline spe1 (purple data) & 53239.0 & $9.6 ; 24.4$ & Soft \\
spe2 (green data) & 53241.2 & $66.1 ; 25.9$ & Hard/Intermediate \\
spe3 (red data) & 53431.2 & $116.1 ; 65.5$ & Hard \\
\hline
\end{tabular}




\section{The spectral evolution}

Spectral analysis was performed by collecting the data corresponding to the same spectral state as shown in the Hardness-Intensity diagram of Figure 2.

\subsection{Soft state}

For the spe1 data (see Table 1) the best fit model is represented by a black body model (or also simple black body model) [6] plus a Comptonization model [7] with parameters showed in Table 2. Changing the diskbb model with the simple black body model the fit doesn't change.

The source shows this spectral state during the soft "outbursts" clearly evident in the JEM-X light curve, when it isn't detected above $30 \mathrm{keV}$.

The spectrum, model and residuals are showed in Figure 3. The unabsorbed bolometric luminosity during this spectral state corresponds to $1.8 \times 10^{38} \mathrm{ergs} \mathrm{s}^{-1}$, i.e. $L / L_{E d d}=0.9$ (assuming a source distance of $9.5 \mathrm{kpc}[8])$.

Table 2: Spectral fitting results for the JEM-X and IBIS broad-band spectra of $4 \mathrm{U} 1722-30$. The model is CompTT for spe3 and CompTT + diskbb for spe1 and spe2.

\begin{tabular}{l|ccc}
\hline \hline parameters & spe1 & spe2 & spe3 \\
\hline$k T_{0}(\mathrm{keV})^{a}$ & 0.40 & 1.33 & 0.81 \\
$k T_{\mathrm{e}}(\mathrm{keV})$ & $2.21_{-0.09}^{+0.20}$ & $11.37_{-0.56}^{+1.31}$ & $40.36_{-15}^{+47.03}$ \\
$\tau$ & $9.06_{-3.40}^{+9.65}$ & $1.33_{-0.09}^{+0.19}$ & $0.48_{-0.35}^{+0.42}$ \\
norm $_{\text {CompTT }}$ & $0.46_{-0.18}^{+0.09}$ & $1.19_{-0.42}^{+0.27} \times 10^{-2}$ & $3.79_{-3.79}^{+2.78} \times 10^{-3}$ \\
\hline$k T_{\text {in }}$ & $0.46_{-0.15}^{+0.10}$ & $0.54_{-0.02}^{+0.02}$ & - \\
norm $_{\text {diskbb }}$ & $2.13_{-0.42}^{+4.93} \times 10^{4}$ & $1.42_{-0.32}^{+0.40} \times 10^{3}$ & - \\
\hline$\chi_{r}^{2}(\mathrm{~d} . o . f)^{b}$ & $1.27(30)$ & $1.03(41)$ & $0.70(55)$ \\
\hline$F_{4-20 \mathrm{keV}}{ }^{b}$ & $2.8 \times 10^{-9}$ & $8.1 \times 10^{-10}$ & $6.4 \times 10^{-10}$ \\
$F_{20-200 \mathrm{keV}}$ & $3.7 \times 10^{-11}$ & $1.5 \times 10^{-10}$ & $3.3 \times 10^{-10}$
\end{tabular}

${ }^{a}$ Fixed parameters

${ }^{b}$ The Fluxes are in units of $\mathrm{erg} \mathrm{s}^{-1} \mathrm{~cm}^{-2}$

\subsection{Hard and Hard/Intermediate state}

The green and red spectral data sets corresponds to the hardening of the source. The Intermediate state is detected just after the soft "outburst" shown in the light curve, and the Hard state follows soon after this.

The Hard/Intermediate state is well represented by the diskbb model plus a Comptonisation model. For the Hard state, the best fit is a simple Comptonisation and the fit doesn't improve by adding a diskbb component. The plasma temperature rises with the hardening, while the optical depth decreases, as indicated in Table 2.

In the Hard state the plasma temperature is not constrained very well and there is the indication of a lack of cut-off, similarly to the Hard state of the atoll 4U 1608-522 [9]. A reflection component is not necessary. The spectra, model and residuals are shown in Figure 3 (panel spe2, spe3). The 
bolometric luminosity of the Intermediate state corresponds to $1.2 \times 10^{38} \mathrm{ergs} \mathrm{s}^{-1}$, that yields a 0.6 $L_{\text {Edd }}$. The bolometric luminosity of the Hard state corresponds to $1.4 \times 10^{37} \mathrm{ergs} \mathrm{s}^{-1}$, i.e. a $L_{\mathrm{Edd}}$ ratio of 0.07 .
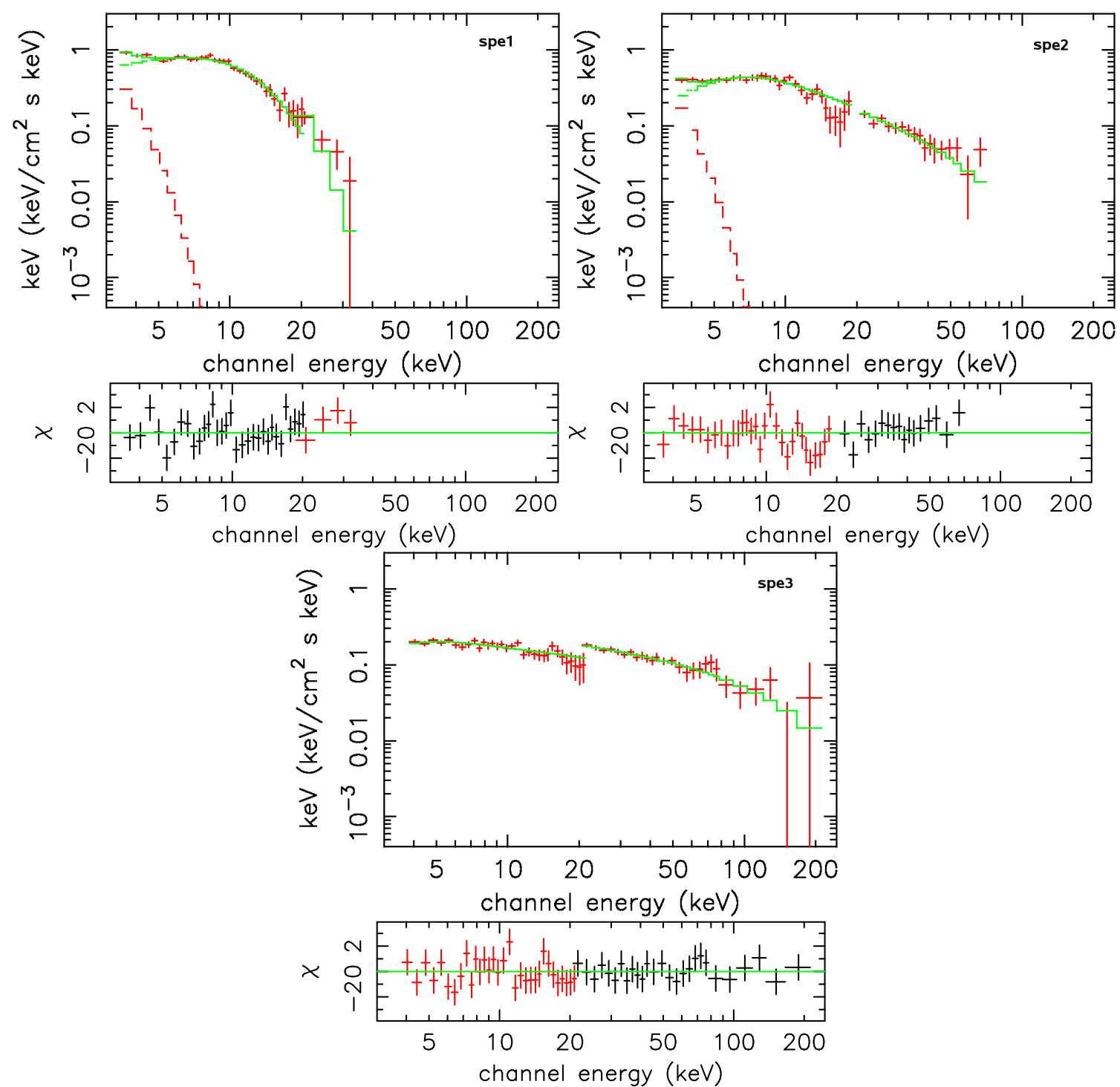

Figure 3: IBIS and JEM-X energy spectra of $4 \mathrm{U} 1722-30$, with the residuals of the data to the model. Spe1 corresponds to the Soft spectral state; spe2 to the Hard-Intermediate; spe3 to the Hard. The models applied are the blackbody plus a Comptonization for spe1 and spe 2 data sets and only a Comptonization for spe3. The best fit parameters are reported in Table 2.

\section{Discussion and conclusion}

The INTEGRAL observations of $4 \mathrm{U} 1722-30$ allow us to follow the X-ray behaviour of this source that is very similar to a X-ray transient, though a real "quiescent" state is never reached. The outbursts are clearly visible in the INTEGRAL light curves, with spectral changes typical 
of transient sources as also confirmed by the color-intensity diagrams, such as the ones for the transient source $4 \mathrm{U}$ 1608-522 [9].

At high soft flux level the source was in Soft state, followed by a hardening. During the Soft state the source doesn't show emission above $30 \mathrm{keV}$, and the spectrum is well described by a cold and optically thick Comptonized corona ( $\tau \sim 9$ and $\mathrm{kT}_{\mathrm{e}} \sim 2 \mathrm{keV}$ ) plus a soft black body emission $\left(k T_{\text {in }} \sim 0.46 \mathrm{keV}\right)$ coming from either the accretion disk or the neutron star.

During the hardening (at low accretion rate) the contribution of the soft component decreases, with a corresponding increase of the hard X-ray emission (up to $200 \mathrm{keV}$ ) described by a hot and optically thin Comptonizing corona ( $\tau \sim 0.5$ and $\mathrm{kT}_{\mathrm{e}} \sim 40 \mathrm{keV}$ ), without any evidence of an energy cut-off.

We estimated the inner radius of the accretion disk in the soft and hard/intermediate state and derived an increase from 5 to $20 \mathrm{~km}$, suggesting an extension of the inner radius during the hardening as also shown by the transient source 4 U 1608-522 [9].

\section{References}

[1] Grindlay, J. E., Marshall, H. L., Hertz, P., et al. ApJ 240, L21,1980

[2] Swank, J.H., Becker, R. H., Boldt, E. A., et al. 1977, ApJL 212 L73.

[3] Olive, J.F., Barret, D., Boirin, L. et al. 1998 A\&A 333, 942

[4] Barret, D., Olive, J.F., Boirin, L. et al. A\&A 357, L41, 1991

[5] Parmar A.N., Stella, L. \& Giommi, P., A\&A 222, 96, 1989

[6] Mitsuda, K. et al., PASJ, 36, 741, 1984

[7] Titarchuk, L. 1994, ApJ 434, 570

[8] Kuulkers, E., den Hartog, P. R., in't Zand, J. J. M., Verbunt, F. W. M., Harris, W. E., \& Cocchi, M., 2003, A\&A, 399, 663

[9] Tarana A., Bazzano A., Ubertini P. ApJ 688, 1295, 2008. 